\title{
Reliable Message Delivery Scheme to Transmit Safety-Message in Heterogeneous VANETs
}

\author{
Joosang Youn \\ Dept. of Industrial ICT Engineering, Dong-Eui University \\ 176, Eomgwangno, Busan jin-gu, Busan, 47340, South Korea \\ jsyoun@deu.ac.kr
}

\begin{abstract}
Recently, various network researches related on self-driving connected cars is studied to support enhanced network performance. These researches have focused on vehicle to vehicle (V2V) technology and vehicle to infrastructure (V2I) technology based on IEEE $802.11 p$ standard. However, in networks employing IEEE $802.11 p$ only, the broadcast storm problem of flooding message and the disconnection problem of network at high and low vehicle densities, respectively. To overcome these problems, this paper proposes dual-interface based reliable message delivery scheme to transmit safety message and cooperative control message in heterogeneous vehicle environments, which combine IEEE 802.11p-based multi-hop VANET and LTE-based cellular VANET. The proposed scheme is progressed by a stable communication network selection (SCNS) scheme which is able to disseminate safety message and cooperative control message with stable reliability.
\end{abstract}

Keywords: VANET, Safety Message, Self-driving, Reliability

\section{Introduction}

Recently, several communication researches related on self-driving connected vehicle is studied to support enhanced network performance. These researches have focused on vehicle to vehicle (V2V) technology and vehicle to infrastructure (V2I) technology and expected to revolutionize the automotive industry in the near future $[1,2,3,4]$. Self-driving connected vehicles are nowadays the area of extensive research. Many companies from automotive and ICT industries try to build their own working for providing self-driving connected cars with enhanced network performance. Communication methods for vehicle networks is based on IEEE $802.11 \mathrm{p}$ standard, which is wireless access for vehicle environments. In networks employing IEEE $802.11 \mathrm{p}$ only, the broadcast storm problem of flooding message and the disconnection problem of network at high and low vehicle densities, respectively, degrade the delay and delivery ratio of safety message dissemination. Therefore, an alternative to the IEEE 802.11p-based VANET, LTE-cellular system based VANET has been investigated due to their low latency, high reliability and wide-range communication. However, a cellular system based VANET communication is not feasible due to the high overhead of communication for message delivery between the vehicles through the base stations and the high number of handoff occurrences through the high mobility of the vehicles. This paper proposes dual-interface based reliable message delivery scheme for safety message and cooperative control message dissemination in heterogeneous vehicle environments, consisting of cooperative self-driving connected cars with dualnetwork interface, which combine IEEE 802.11p-based multi-hop VANET and LTE-

Received (May 25, 2018), Review Result (July 6, 2018), Accepted (July 11, 2018) 
based cellular VANET. In particular, the dual-network interface based reliable message delivery (DRMD) scheme is to provide self-driving connected cars with high reliable message delivery ratio (RMDR) and low message delivery delay (LMDD). The proposed DRMD scheme is progressed by a stable communication network selection (SCNS) scheme which is able to disseminate safety message and cooperative control message with stable reliability. SCNS is based on a novel approach. The feature of SCNS selects the communication network with the relative mobility metric, average hop-counter between cooperative vehicles and the network resource utility for message dissemination. And this scheme considers the cooperative feature of self-driving connected cars. Also, SCNS considers the consumption of network resources to disseminate the information of vehicles through periodic control message and to maintain the network structure. These features decrease the number of safety message and control message dissemination while increasing their stability, thus minimizing the usage of the cellular architecture. To evaluate the performance of DRMD scheme, various key metrics of interest, including RMDR, LMDD, control overhead, and stability, is used. In addition, we show the performance of the proposed scheme compared with both previously proposed schemes, via extensive simulations in NS-3 combined with varied vehicles traffic model and density. The extensive simulation results demonstrate that the proposed scheme shows a stability performance in terms of RMDR and LMDD for safety message and cooperative control message dissemination. The remainder of the paper is structured as follows. The network model is presented in the next Section. In Section 3 we define the proposed dualinterface based reliable message delivery at network-level. Finally, a performance evaluation is provided in Section 4, whereas conclusive remarks are drawn in Section 5 .

\section{Network Model}

In this paper, the network model is assumed as shown in Figure 1. In the assumed network model, PMIPv6 protocol as network protocol is used to support the handover of the mobile vehicle. Also, the mobile vehicle has two network interfaces. One is LTE based $4 \mathrm{G}$ interface and the other is IEEE $802.11 \mathrm{p}$ based WLAN network interface. all network interfaces are active simultaneous.

\section{LTE-based cellular VANET}

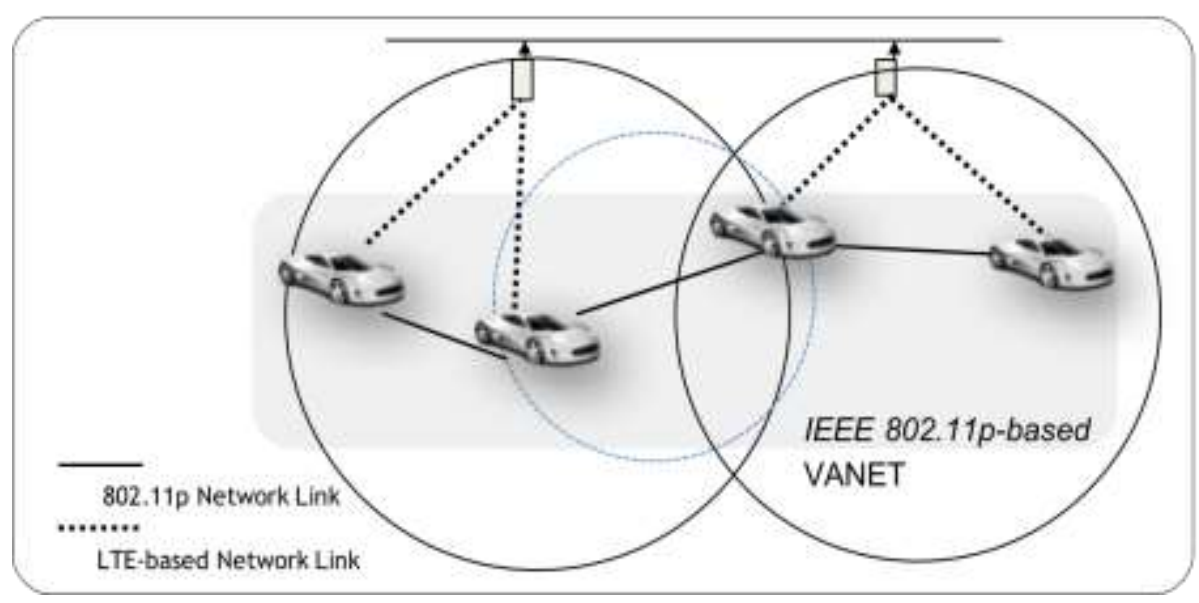

Figure 1. Network Model 


\section{Reliable Message Delivery Scheme}

In this section, dual-interface based reliable message delivery (DRMD) scheme is proposed to delivery safety message and cooperative control message in heterogeneous vehicle environments consisting of cooperative self-driving connected cars with dual-network interface, which combine IEEE 802.11p-based multi-hop VANET and LTE-based cellular VANET. The assumed network can provide a vehicle with QoS scheme for message delivery through DRMD. Also, applications with E2E flows for message delivery are able to require E2E bandwidth $(B)$ to the heterogeneous vehicle environments. Thus, for supporting this, through DRMD, a vehicle searches an E2E path that satisfies $B$ to deliver a control message and safety message during E2E path discovery procedure. In DRMD, the process of path discovery is different per network in a heterogeneous VANET. The following subsection describes DRMD in detail.

\subsection{Vehicle Set}

In DRMD, each vehicle construes the vehicle set (VS) that consists of the onehop, two-hop and three-hop neighbor table as shown in Figure 2. The reason to establish VS is to check that the destination vehicle is located in the cell of the cellular system based BS. Also, it is used for path discovery using admission control in IEEE $802.11 \mathrm{p}$-based multi-hop VANET. In order to find E2E path that satisfies $B$ in IEEE 802.11p-based multi-hop VANET, in our scheme, admission control scheme is used. The admission control scheme is proposed in [5]. [5] studied a source vehicle discover E2E path that satisfies $B$ with low complexity in IEEE 802.11p-based multi-hop VANET. Thus, in DRMD, a vehicle makes the admission decision during E2E path discovery, it will obtain the number of contention links within its interference range by VS. In DRMD, in order to establish VS, the hello message is used. In other word, the information of one-hop neighbor vehicles can be got directly by the hello message. However, there is no way to get the information of neighbor vehicle with more than two-hop directly. Also, a vehicle does not know that whether or not a neighbor vehicle is in the same cell of the LTE-based network
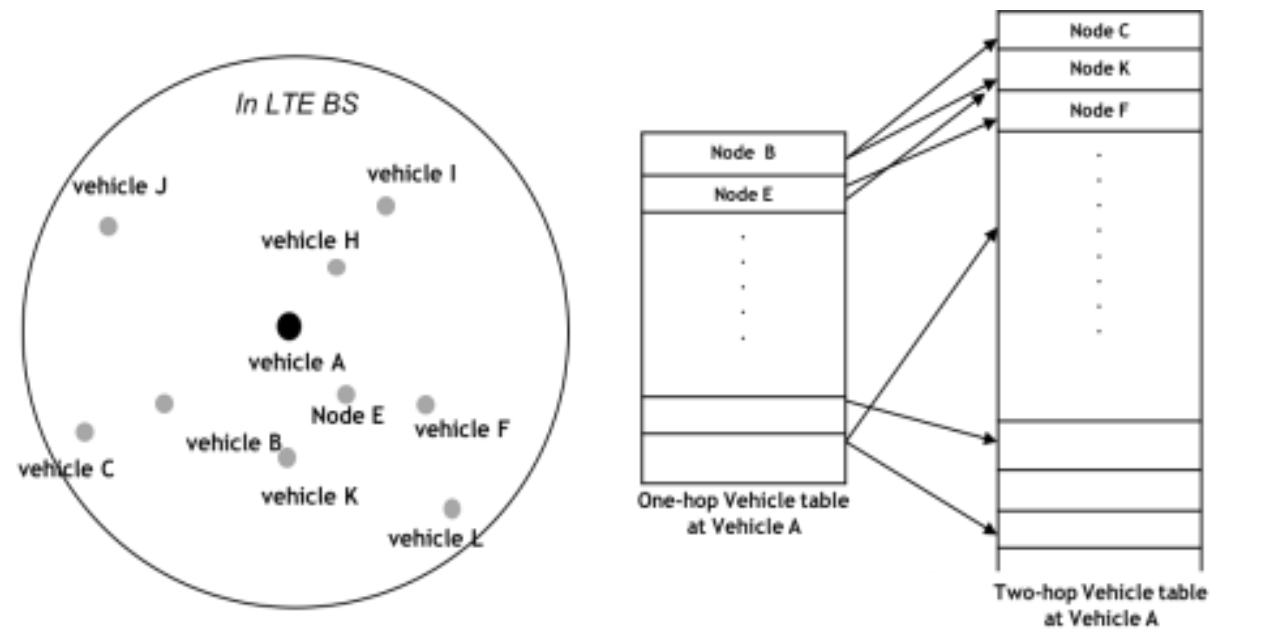

Figure. 2. Vehicle Set at Vehicle A

BS. Thus, when each vehicle construes LCS, it uses a LTE-based cellular vehicle. Thus, if the LTE-based network BS ID of its neighbor vehicle and own the LTEbased network BS ID is different, a vehicle eliminates the information of the neighbor vehicle in LCS. Also, VS is used to discover E2E path that satisfies $B$ in 
IEEE $802.11 \mathrm{p}$-based multi-hop VANET. As mentioned above, neighbor vehicle with more than two-hop count in the VS can be contention vehicle in the interference range when transmitting intra-flow. Thus, in DRMD, The VS is used in the process of E2E path discovery in IEEE 802.11p-based multi-hop VANET. The VS is configured as following. When each vehicle receives the hello-message generated by its one-hop neighbor vehicle, it construes one-hop neighbor table. At this time, the hello message contains the LTE-based network BS ID of the connected LTEbased network interface. Thus, with the LTE-based network BS ID, each vehicle decides whether or not the neighbor vehicle is included in same the LTE-based network BS. If the LTE-based network BS ID is different, a neighbor vehicle is removed in the VS. After estimating the one-hop neighbor table in the VS, each vehicle broadcast hello message with the one-hop neighbor table. And each vehicle construes the two-hop neighbor table. Each vehicle can determine the information of two-hop neighbor vehicle through the hello message received from its one-hop neighbor vehicle. The procedure is updated periodically. In the VS, building threehop neighbor table also performs procedure when receiving the two-hop neighbor table.

\subsection{E2E Path Discovery in LTE-based based VANET}

In order to discover E2E path satisfying B in LTE-based VANET, a vehicle exchanges probe message with the destination vehicle through LTE network interface. At this time, the size of probe message is made with the same amount of B. Also, a vehicle measures RTT with probe message.

\subsection{E2E Path Discovery in IEEE 802.11p-based VANET}

In IEEE 802.11p-based multi-hop VANET, discovering E2E path that satisfies B should be determined. Because in IEEE 802.11p-based multi-hop VANET a vehicle cannot transmit and receive data simultaneously. In other words, to transmit a packet on a one-hop path, the same amount of bandwidth is needed at the sending side and the receiving side. Also, $\mathrm{B}$ is affected according to the location of the vehicle, i.e., source, intermediate and destination vehicle require different local bandwidth [3]. Therefore, in this works, B should be estimated in a per-hop basis. If the same packet was transmitted over a multi-hop path, the required bandwidth is cumulative. Also, the accumulative B is different according to whether or not the vehicle, which receives the packet, transmits the same packet towards a vehicle of destination and the number of contention links in the interference range. In our work, we use the achievable bandwidth for being able to transmit packet in terms of MAC throughput. From [3], in IEEE 802.11p-based multi-hop VANETs, the throughput, $f(x)$, of an E2E path can be described according to the hop number on the E2E path, h, as

$$
f(x)=\left\{\begin{array}{l}
\frac{\min \text { _chanel_bandwidth, }}{\text { min_chanel_bandwidth }}, h=1 \\
\frac{\text { min_chanel_bandwidth }}{3}, h=3 \\
\frac{\text { min_chanel_bandwidth }}{4}, h \geq 4
\end{array}\right.
$$

The equation (1) is based on the analysis in [3]. To get accurate available MAC throughput, we use two parameters, such as the available channel time and the average MAC forwarding delay. To estimate the available bandwidth of IEEE 
802.11p network interface in a vehicle, each vehicle has to determine how much is the free time of channel by listening to the channel at a measurement time. The free time of channel is the available time of channel for a vehicle. We estimate the measurement time ( $\left.\mathrm{T}_{\text {mea_time }}\right)$ that is the same as the default broadcast interval of a control message. Carrier-sense time can be used to determine both free channel time ( $\left.\mathrm{T}_{\text {ava_chann_time }}\right)$ and busy channel time ( $\left.\mathrm{T}_{\text {busy_time }}\right) . \mathrm{T}_{\text {ava_chann_time }}$ should be the remaining allocable bandwidth for the vehicle during the measurement time. An available local bandwidth is determined with available channel time and average MAC forwarding delay during the measurement time in the forwarding queue of $802.11 \mathrm{p}$ network interface in a vehicle. The average MAC forwarding delay is defined as the average time from the time when a new arrival packet is in a forwarding queue of a vehicle to the time when the vehicle receives the successful transmission of the packet at MAC layer. So, the MAC forwarding delay, $\mathrm{T}_{\text {mac_forwarding_delay, }}$ is shown

$T_{\text {mac_forwarding_delay }}=T_{\text {ack }}-T_{\text {queue }}$

In this paper, the average value of the forwarding time taken to complete transmission of a packet, including MAC access delay is used to access channel and estimate the time of retransmission. The weighted moving average is used to smooth the estimated MAC forwarding delay of a queue. After the forwarding of each packet is completed, the value is updated as

$\bar{T}_{\text {mac_forwarding_delay }}=\alpha \bar{T}_{\text {mac_forwarding_delay }}+(1-\alpha) T_{\text {mac_forwarding_delay }}$

where $\mathrm{T}_{\text {mac_forwarding_delay }}$ is the average value of the previous packet, is the weighting factor $(<1)$, whose optimum value has been computed to be 0.9 , following a comprehensive simulation under traffic conditions, and $\mathrm{T}_{\text {mac_forwarding_delay }}$ is the forwarding delay gotten by the packet. The MAC forwarding delay also includes the time for the head-of-line packet to be transmitted to the physical layer. This means that the overhead of the transmission in the contending area is included. In particular, the period for successful control message exchange is included, if this exchange is used for packet transmission. With the average MAC forwarding delay and available channel time, we can estimate the expected number of packets, $\mathrm{N}$, that can be transmitted during the next measurement period. Thus, $\mathrm{N}$ is determine d, as

$$
N=\left(\frac{T_{\text {free_channeltime }}}{\overline{T_{\text {mac_forwarding_delay }}}}\right)
$$

where $\mathrm{N}$ is the number of packets that are transmitted during next measurement time. Using the value, we are able to predict the available bandwidth as

$$
B_{\text {avaliable }}=\left(\frac{N \times P L}{T_{\text {mea_time }}}\right)
$$

where PL is any MAC layer payload length transmitted in the current measurement time. In this paper, the scheme, for discovering E2E path which satisfies B in IEEE 802.11p-based VANET, is modified with the admission control scheme in [5]. In IEEE 802.11p-based VANET, the whole procedure for discovering E2E path that satisfies B is progressed when disseminating a control packet during 
an E2E path discovery. To initiate an E2E path discovery, a source vehicle disseminates a control packet with B. At this time, the source vehicle firstly checks whether there is a destination IP in the one-hop neighbor table and the two-hop neighbor table in the VS. Through this procedure, it can determine whether the E2E hop counter is 1-hop or more than and whether or not the connectivity in the VS is possible. $\mathrm{B}$ is determined with the hop counter of E2E obtained by the above procedure. At an intermediate vehicle and a destination vehicle, B is also determined with this procedure and the hop-counter achieved by the received control packet. Figure 3 shows the pseudo codes for an E2E path discovery procedure by handling a control message at each vehicle. Here, $B a, i$ is the available bandwidth of vehicle $\mathrm{i}$ and Hop is the E2E hop counter achieved by the current control message.

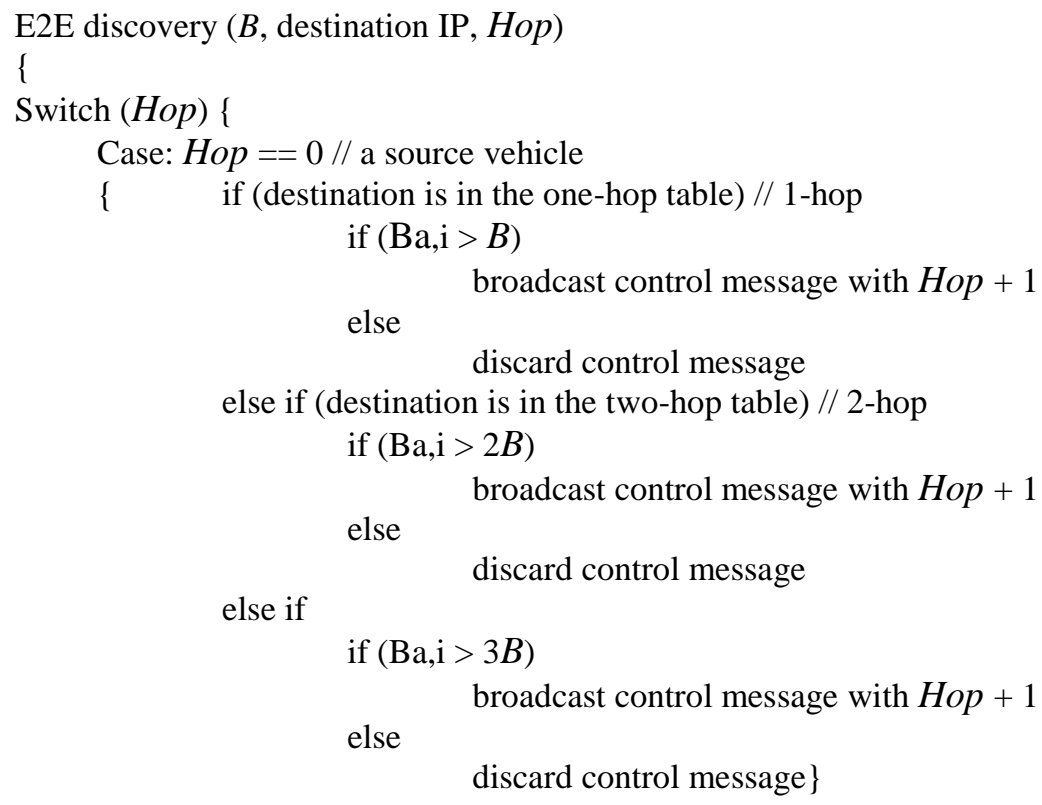

Case: $H o p==1 / /$ an intermediate vehicle

$\{\quad$ if (this vehicle is a destination) // 1-hop

if $(\mathrm{Ba}, \mathrm{i}>B)$

transmit control message to a source vehicle

else discard control message

else if (destination is in the one-hop table) // 1-hop

if $(\mathrm{Ba}, \mathrm{i}>2 B)$

broadcast control message with $H o p+1$

else

discard control message

else if //end-to-end hop number is more than 2-hop

if $(\mathrm{Ba}, \mathrm{i}>3 B)$

broadcast control message with $H o p+1$

else

discard control message\}

Case: $H o p==2 / /$ an intermediate vehicle

\{ if (this vehicle is a destination) // 2-hop

if $(\mathrm{Ba}, \mathrm{i}>2 \mathrm{~B})$

transmit control message to a source vehicle

else 


\author{
discard control message \\ else if // more than 2-hop \\ if $(\mathrm{Ba}, \mathrm{i}>3 B)$ \\ broadcast control message with Hop +1 \\ else \\ discard control message \} \\ \}
}

\title{
Figure 3. The Pseudo Codes for E2E Discovery
}

\subsection{Communication Network Selection Decision}

A communication network selection decision procedure is efficiently carried out at a vehicle with the following two-step. In the first step, whether or not a connectivity between a source vehicle and a destination vehicle is possible in the IEEE $802.11 \mathrm{p}$-based network interface. Thus, a vehicle has to determine whether or not there is a destination vehicle in VS. The process of determining that the connectivity is possible is as following. Firstly, a source vehicle finds the destination vehicle in VS. If there is not a destination vehicle in VS, a vehicle transmits a data through IEEE 802.11p-based network without the communication network selection decision procedure by DRMD. If there is a destination vehicle, a vehicle sends the control message with $B$ to an ad-hoc network side through IEEE 802.11p-based network interface in order to discover E2E path satisfying B. Also at the same time it sends the probe request message generated with B to IEEE $802.11 \mathrm{p}$ based network side through IEEE $802.11 \mathrm{p}$-based network interface. Thus, through this step, a vehicle can obtain multi-E2E path satisfying B in VANET. The second step is the process for making a radio interface decision. A vehicle waits to two response messages about two request messages. If a vehicle receives the probe response message from the IEEE 802.11p-based network side and does not receive control message from an ad-hoc network side, as a primal network for transmitting a data, the IEEE 802.11p-based network is determined. Whereas if a vehicle receives the probe response message and the control message, it must make a communication network selection decision for a network. In DRMD, if there are multi-E2E paths satisfying B in VANET, an IEEE 802.11p-based network is set as a default network, because there is an E2E path satisfying B in an IEEE 802.11p-based network.

\section{Performance Evaluation}

To evaluate the performance of the proposed DRMD, the performance of the proposed DRMD is evaluated and compared with IEEE 802.11p-based network environment in two scenarios; grid topology and static random topology. In the simulations, in order to consist of the dual-network based VANET environments, we use LTE-based cellular VANET and the IEEE $802.11 \mathrm{p}$-based VANE. Simulations are conducted using the ns-3 simulator. The dual-network based VANET environments are considered using random topologies as shown in Figure 4, where 16 nodes with dual network interfaces are located in $1000 \mathrm{~m} \times 1000 \mathrm{~m}$ square regions. There are 20, 30, 40 flows which have different transmission rate and starting time. The packet size of all flows is 2000-byte and through varying packet interval per packet, the transmission rate of each flow is controlled. The metrics used to measure the performance are the average data transmission delay. The simulation runs for 200s. Network topology used in the simulation is shown in Figure 4. 


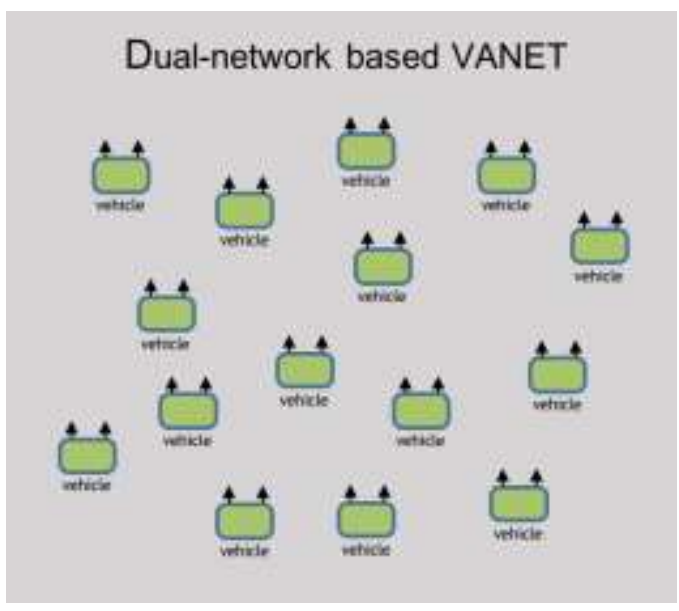

Figure 4. Random Topology used to Evaluate the Performance

Two simulations are evaluated in this scenario. One is evaluated in only LTE-based VANET. The other is evaluated in the dual-network based VANET environment using DRMD. The simulation results are shown in Figure 5. There shows the performance improvement in the results of the simulation with all nodes that are enabled to use DRMD, compared with the results in the simulation on single network using only LTEbased network interface. In the results, all admitted flow is provided with stable transmission service through flow. This shows that DRMD can distribute data transmission through dual-network based VANET.

In addition, in this works, the stability of data transmission delivery, $\mathrm{S}$, is defined to

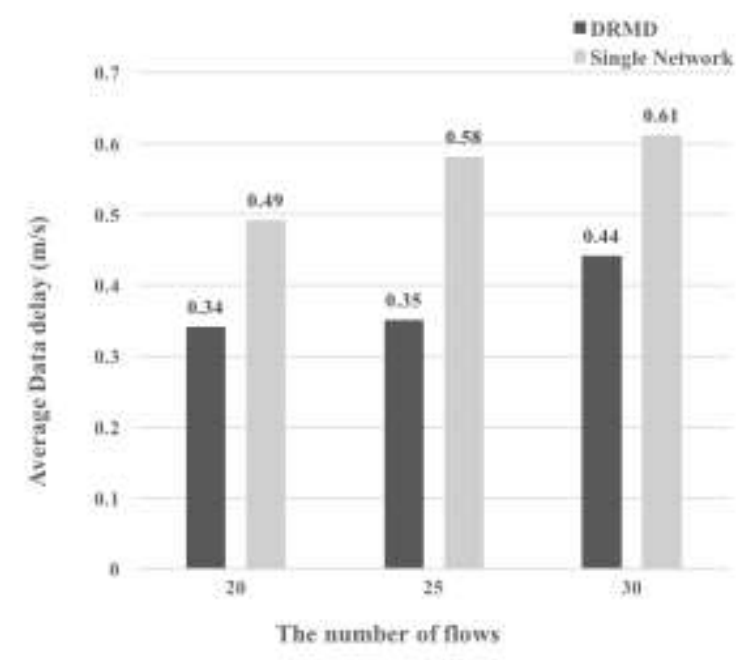

Figure 5. Average Data Transmission Delay in the Random Topology

evaluate reliable message delivery ratio (RMDR) and low message delivery delay (LMDD) with the total percentage of data loss during the data transmission and data transmission delay. When the percentage loss is less than $5 \%$, the level of service quality is good but when percentage loss is between $5 \%$ and $10 \%$, quality is medium. When percentage loss exceeds $15 \%$, quality is poor. We obtain good quality $(\mathrm{S}=3.4 \%)$, when using DRMD. However, when vehicle uses only LTE network interface in on LTE-based VANET, low quality ( $\mathrm{S}=14 \%$ ) is obtained. By comparison, DRMD gives better stable service than the scheme using only IEEE 802.11p network interface. In addition, the results show that DRMD gets better data transmission service in terms of the stability of 
data transmission delivery. In order to evaluate more realistic performance of DRMD, the simulations run in the dual-network based VANET environments where 25 mobile vehicles with mobility are located randomly and have mobility in $1000 \mathrm{~m} \times 1000 \mathrm{~m}$ square regions. The simulations are evaluated in two same scenarios above. One is evaluated in only LTE-based VANET. The other is evaluated in the dual-network based VANET environments using both LTE network interface and IEEE $802.11 \mathrm{p}$ network interface. In the simulations, there are 3-flows with E2E bandwidth requirement of $0.2 \mathrm{Mbps}$, 3-flows with E2E bandwidth requirement of $0.25 \mathrm{Mbps}$ and 4-flows with E2E bandwidth requirement of $0.3 \mathrm{Mbps}$. The E-2-E pair is chosen randomly. The simulations go for 300s. In the simulations, the metrics used in measuring the throughput utility. The throughput utility is min (1, Tact/Treq). Treq is the upper bound throughput of bandwidth requirement and Tact is the measured throughput. Fig. 6 shows the throughput utility, as simulation results. The LTE-based VANET shows the low throughput utility, while DRMD gets the high throughput utility. In the other words, the scheme using DRMD also shows better a stable data transmission than scheme using only LTE network interface in LTE-based VANET.

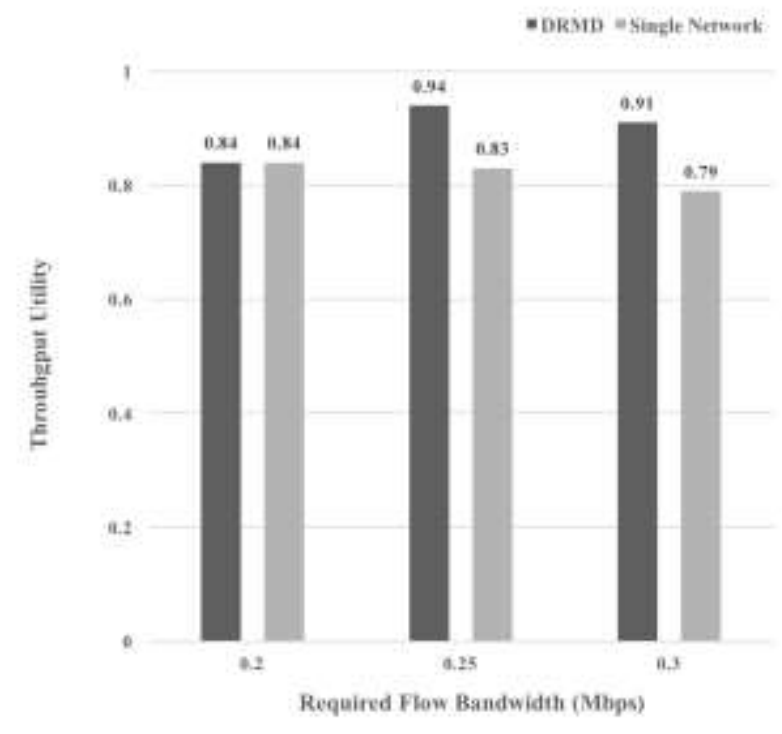

Figure 6. The Throughput Utilities in the Random Topology

\section{Conclusion}

In heterogeneous environment, a vehicle with dual-network interfaces has the ability to attach to multiple networks simultaneously. In this paper, a novel dual-network interface-based communication network selection scheme is proposed to guarantee E2E bandwidth demanded for the efficient transmission of a control and safety message. In this work, through the proposed DRMD, a node estimates the current network status during path discovery for message delivery and then determines the network interface to transmit message. Simulations results demonstrate that DRMD can significantly get the high throughput utility and the high data delivery stability in terms of the control and safety message transmission for self-driving connected cars.

\section{Acknowledgments}

This research was supported by Basic Science Research Program through the National Research Foundation of Korea (NRF) funded by the Ministry of Education (NRF-2017R1D1A1B03034689 and is a revised and expanded version of a paper 
entitled [Communication Network Selection Scheme for Data Dissemination in Heterogeneous VANETs] presented at [AGST 2018, Hanoi, Vietnam, June 28-30, 2018].

\section{References}

[1] S. Chen, J. Hu, Y. Shi, Y. Peng, J. Fang, R. Zhao and Li Zhao, "Vehicle-to-Everything (v2x) Services Supported by LTE-based Systems and 5g", IEEE Communications Standards Magazine, (2017), pp. 7076.

[2] J. Park and D. Yun, "The recognition prioritization of road environment for supporting autonomous vehicle", Journal of the Korea Academia-Industrial Cooperation Society, vol. 19, no. 2, (2018), pp. 595601.

[3] W. Yi, “A New Congestion Control Algorithm for Vehicle to Vehicle Safety Communications", Journal of the Korea Academia-Industrial Cooperation Society, vol. 18, no. 5, (2017), pp. 125-132.

[4] G. Yan and D. B. Rawat, "Vehicle-to-vehicle connectivity analysis for vehicular ad-hoc networks", Ad Hoc Networks, vol. 58, (2016), pp. 25-35.

[5] J. Sang Youn, S. Pack and Y.-G. Hong, "Distributed admission control protocol for end-to-end QoS assurance in ad hoc wireless networks", EURASIP J. Wireless Comm. and Networking, vol. 163, (2011), pp. $1-18$

\section{Author}

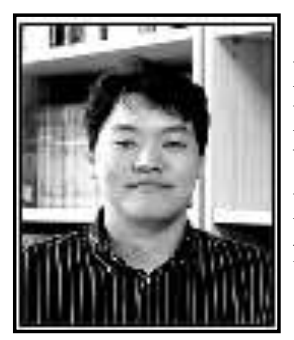

Joosang Youn, is currently associate professor in Dept. of Industrial ICT Engineering at Dong-Eui University since 2008. $\mathrm{He}$ received the B.S., M.S. and Ph.D. degrees from Korea University, Seoul, Korea in 2001, 2003 and 2008, respectively. His current research interests include the IoT, 5G, VANET and future network architecture. 